Efeito da aplicação sônica do adesivo na resistência de união de pinos de fibra cimentado à dentina exposta ao hipoclorito de sódio

\author{
Effect of sonic application of adhesive on the bond strength of fiber posts cemented to dentin exposed to sodium \\ hypochlorite
}

\title{
Juliana Marques
}

Programa de Engenharia Metalúrgica e de Materiais, Universidade Federal do Rio de Janeiro, Rio de Janeiro,Brasil

Marta Dantas

Departamento de Clínica Odontológica, Endodontia, Faculdade de Odontologia, Universidade Federal do Rio de Janeiro, Rio de Janeiro, Brasil

Renata Simão

Programa de Engenharia Metalúrgica e de Materiais, Universidade Federal do Rio de Janeiro, Rio de Janeiro,Brasil

Maira Prado

Programa de Engenharia Metalúrgica e de Materiais, Universidade Federal do Rio de Janeiro, Rio de Janeiro,Brasil

- Os autores declaram que não há conflito de interesse.

\section{Resumo}

Objetivo: 0 objetivo deste estudo foi avaliar o efeitoda aplicação sônica do adesivo na resistência de união de pinos de fibra cimentado à dentina previamente exposta ao hipoclorito de sódio ( $\mathrm{NaOCl}$ ). Material e Métodos: Trinta dentes bovinos unirradiculares foram utilizados. Os dentes foram seccionados produzindo espécimes com 7 $\mathrm{mm}$, referente ao terço cervical e parte do terço médio da raiz. Os canais foram preparados e as amostras divididas em três grupos ( $\mathrm{n}=10)$. No grupo 1 , as raízes foram irrigadas com água destilada e secas com cone de papel. Nos grupos 2 e 3 , as raízes foram imersas em $\mathrm{Na}$ OCle, em seguida, irrigadas e secas como descrito no grupo 1. Para cimentação do pino, nos grupos 1 e 2, o adesivo foi aplicado manualmente e no grupo 3 com o dispositivo sônico Smart Sonic. Após cimentação dos pinos,os espécimes foram segmentados em slicese submetidos ao teste de push-out para análise deresistência de união. Os dados foram avaliados estatisticamente pelos testes Kruskal-Wallis e Mann Whitney ( $p<0,05)$. Resultados: Os resultados de resistência de união expressos em mediana (MPa) foram: Grupo 1 (4,12 MPa) $=$ Grupo $3(3,41$ $\mathrm{MPa}$ ) GGupo 2 (2,71 MPa).Conclusão: Conclui-se que a aplicação sônica do adesivo afetou os níveis de resistência de união do sistema Âmbar/AllCem à dentina exposta ao $\mathrm{NaOCl}$, atingindo valores significativamente semelhantes ao grupo sem $\mathrm{NaOCl}$.

Palavras-chave: cimentação; adesivos; hipoclorito de sódio.

\section{Abstract}

Objective: The aim of this study was to evaluate the effect of sonic application of the adhesive on the bond strength of fiber posts cemented to dentine previously exposed to sodium hypochlorite ( $\mathrm{NaOCl}$ ). Material and Methods: Thirty single-rooted bovine teeth were used. The teeth were sectioned producing specimens with $7 \mathrm{~mm}$, referring to the cervical and part of the middle third of the root. Root canals were prepared and the samples divided into 3 groups $(n=10)$. Group 1 the roots were irrigated with distilled water and dried with paper points. In groups 2 and 3 , the roots were immersed in $\mathrm{NaOCl}$ and then irrigated and dried as described in group 1. For post cementation, in groups 1 and 2 the adhesive was applied manually and in the group 3 with the sonic device Smart Sonic. After cementation,the specimens were segmented into slices and submitted to the push-out test for bond strength analysis. Data were statistically analyzed by Kruskal-Wallis and Mann-Whitney tests $(p<0.05)$. Results: The bond strength results expressed as median (MPa) were: Group $1(4.12 \mathrm{MPa})=$ Group $3(3.41 \mathrm{MPa})>$ Group 2 (2.71 MPa). Conclusion: In conclusion, the sonic application of adhesive affected the bond strength levels of Ambar/Allcem system to dentin exposed to $\mathrm{NaOCl}$, reaching values significantly similar to the group without $\mathrm{NaOCl}$.

Keywords: cementation; adhesives; sodium hypochlorite.

\section{Introdução}

D evido à extensa perda de estrutura dental, seja por cárie ou trauma, para restauração de dentes tratados endodonticamente,frequentemente faz-se necessário a utilização de um retentor intrarradicular, associado a um cimento resinoso e um compósito. ${ }^{1}$

Durante o preparo químico-mecânico, instrumentos endodônticos são utilizados em associação com substâncias químicas auxiliares. Dentre essas substâncias, o hipoclorito de sódio $(\mathrm{NaOCl})$ é, mundialmente, o irrigante mais utilizado por associar atividade antimicrobiana e capacidade de dissolução tecidual. ${ }^{2,3}$ Entretanto,devido a sua ação proteolítica, o Na$\mathrm{OCl}$ danifica a estrutura colágena da dentina e reduz a microdureza dentinária. ${ }^{4,5}$ Além disso, é um forte agente oxidante. Durante o seu uso, há a formação de uma camada de oxigênio livre na superfície dentinária que inibe a polimerização de materiais resinosos, afetando negativamente o processo de adesão desses materiais à dentina. ${ }^{4-7}$

Estudos prévios mostraram que a falha na adesão dos pinos ocorre principalmente na interface cimento resinoso/dentina.$^{8-10}$ Ainda é relatado um efeito deletério do $\mathrm{NaOCl}$ na resistência de união dos agentes cimentantes dos pinos de fibra à dentina. Diferentes estratégias têm sido sugeridas para aumentar a performance dos sistemas adesivos,entretanto tais estratégias estão geralmente associadas ao aumento de passos clínicos e os resultados encontrados na literatura são controversos. ${ }^{8-10}$

Mena-Serrano et al. mostraram que a aplicação do adesivo com um dispositivo sônico melhorou a resistência de união de sistemas adesivos à dentina coronal. ${ }^{11}$ Ainda, Cuadros-Sanchez et al. verificaram que a aplicação de adesivo no canal radicular, utilizando um dispositivo sônico, aumentou os níveis de resistência de união de pinos de fibra cimentados à dentina, assim como permitiu uma maior penetração do adesivo. ${ }^{9}$

O objetivo do presente estudo foi avaliar o efeito da aplicação sônica do adesivo na resistência de união de pinos de fibra cimentado à dentina previamente exposta ao hipoclorito de sódio. As hipóteses nulas avaliadas foram: (i) o hipoclorito de sódio não é capaz de alterar os níveis de resistência de união da dentina e (ii) a aplicação sônica do adesivo Âmbar com o dispositivo SmartSonic não influencia nos níveis de resistência de união. 


\section{Material e Métodos}

\section{- Preparo dos Dentes}

Trinta dentes bovinos unirradiculares foram utilizados no presente estudo. Os dentes foram seccionados na junção amelo-cementária com disco de carborundum(Labordental, São Paulo, SP, Brasil) acoplado emmicromotor de bancada (Beltec,Araraquara, SP, Brasil). Um segundo corte foi realizado a fim de padronizar os espécimes em $7 \mathrm{~mm}$, referente ao terço cervical e parte do terço médio da raiz.Posteriormente, para eliminar a conicidade do canal, as amostras foram preparadas com uma broca cilíndrica, com $3 \mathrm{~mm}$ de diâmetro, fixada em uma furadeira de bancada (FG-16, Ferrari, SP, Brazil). As amostras foram divididas aleatoriamente em três grupos $(\mathrm{n}=10)$ :

No grupo 1(grupo controle), as raízes foram irrigadas com $20 \mathrm{ml}$ de água destilada e secas com cone de papel absorvente (Meta Biomed,Horsham, PA, EUA).

Nos grupos 2 e 3, as raízes foram imersas em hipoclorito de sódio 5,25\% (Mil Fórmulas, Rio de Janeiro, RJ, Brasil) por 20 minutos. ${ }^{12}$ Em seguida, foram irrigadas com $20 \mathrm{ml}$ de água destilada e secas com cone de papel absorvente. A cimentação do pino foi realizada, respectivamente,sem e com o auxílio do dispositivo sônico para aplicação do adesivo.

\section{- Preparo do Pino}

Os pinos de fibra de vidro White Post DC3 (FGM, Joinville, SC, Brasil) foram lavados por dez minutos em ultrassom com álcool 70\% para limpeza e remoção de contaminantes e secos com jatos de ar comprimido. ${ }^{13}$ Em seguida, uma fina camada de silano (Prosil, FGM, Joinville, SC, Brasil) foi aplicada na superfície dos pinos com auxílio de um microaplicador (cavibrush, FGM, Joinville, SC, Brasil). Após 1 minuto, foi realizada leve secagem com jato de ar na superfície do pino, conforme recomendações do fabricante.

\section{- Cimentação dos Pinos}

Nos grupos 1 e 2 foram realizados condicionamentos com ácido fosfórico 37\% (Condac, FGM, Joinville, SC, Brasil) por 15 segundos, lavagem e secagem do conduto. Com auxílio de um microaplicador, foi realizada a aplicação do adesivo Âmbar (FGM, Joinville, SC, Brasil) no conduto radicular. Aplicou-se jato de ar por 10 segundos para evaporação do solvente e, em seguida, foi realizada a fotopolimerização através de Led (Radii-cal, SDI, São Paulo, SP, Brasil) por 20 segundos. O cimento AllCem (FGM, Joinville, SC, Brasil) foi introduzido no contudo radicular com auxílio da ponta misturadora, a parte cilíndrica do pino posicionada no centroda raiz e, em seguida, foi realizada fotopolimerização por 40 segundos.

No grupo 3, foi realizado o condicionamento da superfície dentinária e o adesivo foi aplicado com auxílio de uma ponta com formato de um microaplicador, fixada no dispositivo de agitação sônica, Smart Sonic Device (FGM, Joinville, SC, Brasil). Após 5 segundos de aplicação sônica, empregou-se jato de ar e foi realizadapolimerização como descrito anteriormente. O cimento foi introduzido no canal e, em seguida, o pino foi posicionado, como previamente descrito para os grupos 1 e 2 . As amostras foram mantidas em estufa, a $37^{\circ} \mathrm{C}$ e umidade relativa de $100 \%$ por 1 semana.

\section{- Preparação das Amostras para Teste de push-out}

As amostras foram seccionadastransversalmente em uma máquina de corte IsoMet $^{\circledR}$ (Buehler, Lake Bluff, IL, EUA) com disco diamantado (Série 15HC, Buehler, Lake Bluff, IL, EUA), a fim de produzir fatias de, aproximadamente, $1 \mathrm{~mm}$ de espessura. A espessura exata de cada fatia foi determinada com um paquímetro digital (MPI/E-101, Mytutoyo, Tóquio, Japão). Um total de trêsslices foi analisado por amostra, totalizando 30 discos por grupo.

As amostras foram posicionadas em uma base metálica de aço inoxidável contendo um orifício na região central, sendo o pino posicionado na direção do orifício. O conjunto foi posicionado na base da máquina de ensaio universal EMIC DL1000 (São José dos Pinhais, PR, Brasil) com carga de $200 \mathrm{kgf}$. A haste metálica com ponta ativa de $1,2 \mathrm{~mm}$ de diâmetro foi fixada no mordente da máquina e posicionada no centro do pino de fibra de vidro. O ensaio de resistência ao cisalhamento por extrusão (push-out) foi conduzido à velocidade de $0,5 \mathrm{~mm} / \mathrm{min}$. Os valores de resistência da união foram obtidos em newtons $(\mathrm{N})$. A partir disto, a resistência de união, expressada em megapascais $(\mathrm{MPa})$, foi calculada dividindo a força $(\mathrm{N})$ pela área $\left(\mathrm{mm}^{2}\right)$ da interface adesiva, utilizando-se a formula: $\mathrm{A}=2 \pi \mathrm{rh}$, em que: $\pi=3,14 ; \mathrm{r}=$ raio do canal $(1,5 \mathrm{~mm})$; $\mathrm{h}=$ espessura da fatia, em milímetros. ${ }^{13}$ A Figura 1 mostra um desenho esquemático dos diferentes passos do preparo das amostras. 

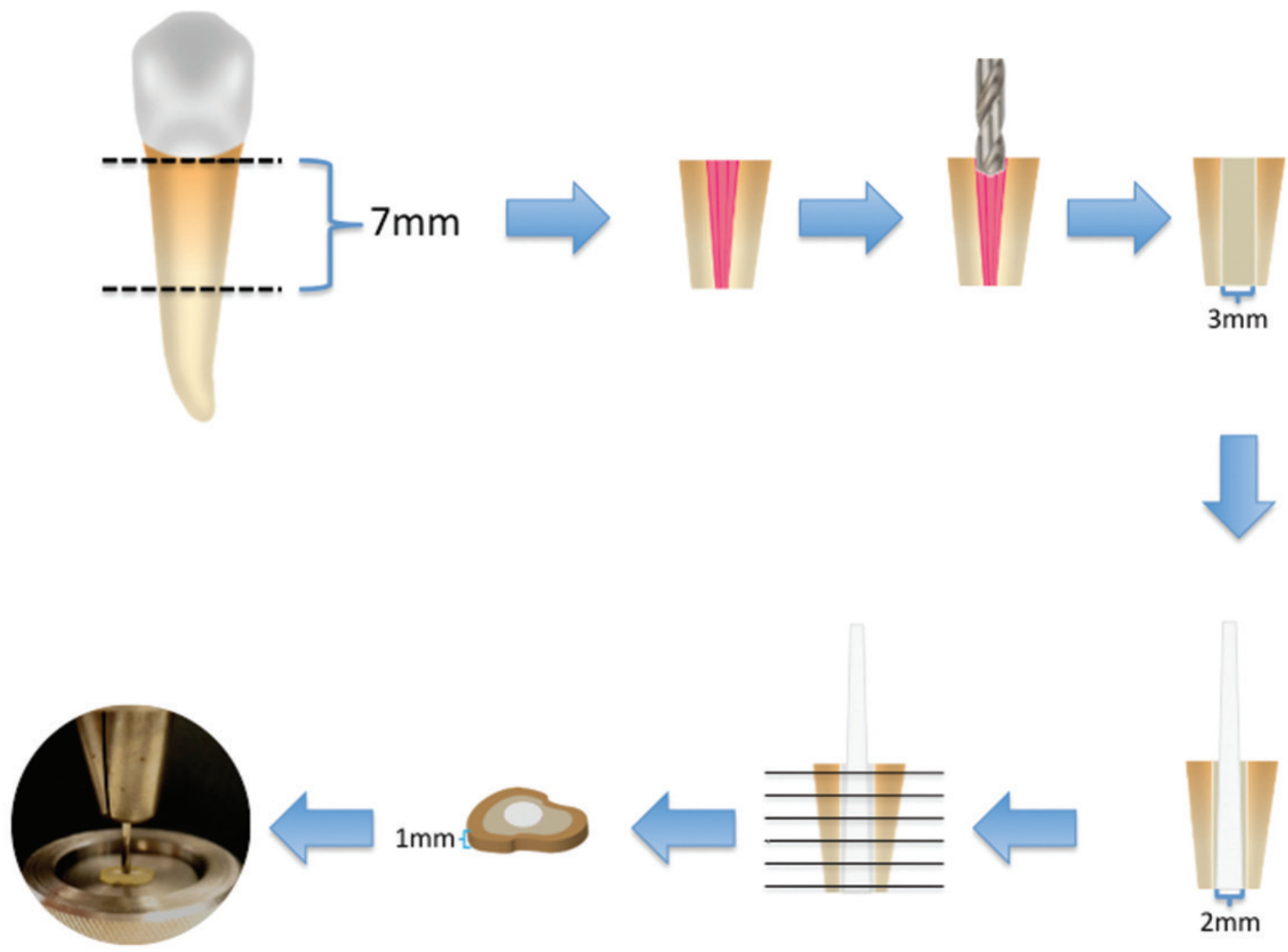

Figura 1. Desenho esquemático dos diferentes passos do preparo das amostras

\section{- Análise do Padrão de Fratura}

A análise da fratura das amostras após o teste de push-out foi realizada em lupa estereoscópica (Nikon SMZ 800, Japão) para determinar o padrão de fratura como: ${ }^{14}$

Tipo I: Adesiva cimento/dentina: a fratura ocorre na adesão, predominantemente na interface cimento/dentina.

Tipo II: Mista coesiva dentina: independente da interface envolvida ocorre alguma fratura na dentina.

Tipo III: Mista coesiva pino: independente da interface envolvida ocorre fratura no pino.

Tipo IV: Mista coesiva pino/dentina: independente da interface envolvida ocorre fratura no pino e na dentina.

\section{- Análise Estatística}

Os dados obtidos de resistência da união foram analisados e submetidos ao teste de normalidade Kolmogorov-Smirnov $(\alpha=0,05)$. Os dados apresentaram distribuição não normal, sendo então submetidos aos testes Kruskal-Wallis e Mann Whitney $(\mathrm{p}<0,05)$.

\section{Resultados}

A Tabela 1 mostra a análise descritiva dos valores de resistência de união dos grupos avaliados. É possível observar que o uso do hipoclorito de sódio levou a uma redução nos níveis de resistência de união do sistema Âmbar/ AllCem à dentina. Entretanto, quando o dispositivo sônico foi empregado, os valores deresistência de união aumentaram, atingindo valores significativamente semelhantes ao grupo 1, controle, sem $\mathrm{NaOCl}$.

Em relação ao padrão de fratura, observa-sena Figura 2 que em todos os grupos avaliados houve predomínio de fratura tipo I, adesiva cimento/dentina. 


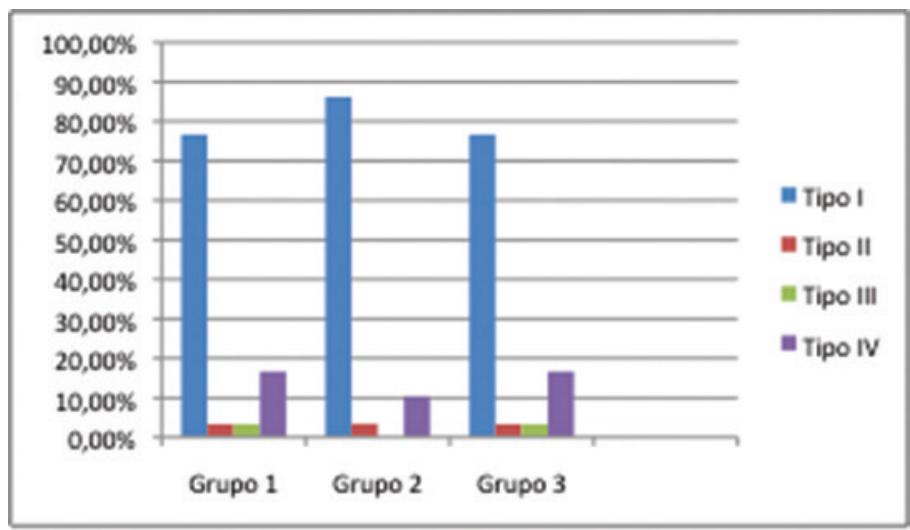

Figura 2. Análise de padrão de fratura (\%)

\section{Discussão}

Atualmente, com os conceitos de Odontologia adesiva, os pinos de fibra de vidro vêm sendo amplamente utilizados devido, entre outras características, à sua estética e propriedades mecânicas. ${ }^{15}$

No presente estudo, foi utilizado dente bovino devido à facilidade na aquisição das amostras, além das similaridades estruturais com a dentina humana em relação à composição e quantidade de túbulos. ${ }^{16-18}$ Saunders avaliou o efeito de quatro agentes de adesão na resistência de união em dentina humana e bovina. $\mathrm{O}$ autor verificou que não houve diferença significativa entre os diferentes substratos em relação aos sistemas testados. A análise em microscopia eletrônica de varredura mostrou que as superfícies dentinárias dos dentes humanos e bovinos apresentavam semelhanças em relação à densidade, diâmetro e orientação dos túbulos dentinários, sugerindo que o uso dos dentes bovinos, como substituto para os dentes humanos. ${ }^{16}$

Em relação ao preparo das amostras, as raízes foram padronizadas em $7 \mathrm{~mm}$, correspondente ao terço cervical e parte do terço médio da raiz. Os pinos de fibra de vidro são cimentados a partir de técnicas adesivas, não havendo a necessidade de retenção em $2 / 3$ da raiz. O objetivo da utilização desses pinos é reter as restaurações coronárias, seja ela direta ou indireta, ao remanescente dental, e distribuir uniformemente as forças mastigatórias a esteremanescente. ${ }^{19}$

No presente estudo os canais radiculares não foram obturados para que apenas o efeito do hipoclorito de sódio fosse avaliado. Estudos prévios mostraram um efeito negativonão apenas dos irrigantes, mas também dos materiais obturadores endodônticos, nos níveis de resistência de união dos materiais resinosos à dentina. ${ }^{5,6,19-21}$

Em relação ao protocolo de irrigação, optou-se pela imersão dos dentes em hipoclorito de sódio 5,25\% durante 20 minutos, simulando o tempo de contato do irrigante com a superfície dentinária durante o tratamento endodôntico. ${ }^{12}$ Com a imersão, os dentes foram colocados e removidos da solução em um mesmo momento, permitindo uma padronização total de tempo. Além disso, empregou-se um volume grande de irrigante devido ao efeito autolimitante do $\mathrm{NaOCl} .{ }^{2}$ Em relação ao uso da água destilada, optou-se pela irrigação com seringa, a fim de permitir o fluxo-refluxo da solução, auxiliando na remoção do $\mathrm{NaOCl}$, além de melhor reproduzir a prática clínica.

A primeira hipótese nula avaliada foi rejeitada, o emprego do hipoclorito de sódio, simulando o tempo do preparo químico-mecânico, afetou negativamente os níveis de resistência de união da dentina. Esses achados corroboram com estudos prévios e estão associados aos danos causados na estrutura colágena, ${ }^{4}$ assim como a formação de oxigênio nascente na superfície dentinária que interfere na polimerização dos materiais adesivos resinosos. ${ }^{20,21}$

A aplicação sônica do adesivo promoveu um aumento dos níveis de resistência de união, apresentando valores estatisticamente semelhantes ao grupo sem hipoclorito de sódio, rejeitando a segunda hipótese nula. A agitação sônica pode ter permitido uma maior penetração do adesivo, associado o um aumento na difusão dos monômeros no interior dos túbulos dentinários, auxiliando ainda na evaporação de solventes. ${ }^{9,11}$

Embora o uso do dispositivo sônico tenha favorecido os níveis de resistência de união, em todos os grupos avaliados, o tipo de fratura predominante foi na interface adesiva cimento resinoso/dentina, mostrando que esta é, ainda, a interface mais frágil neste processo de adesão.

Novos estudos associando o uso do hipoclorito na irrigação e diferentes cimentos na obturação são necessários para avaliar a interação irrigante cimento e este efeito associado ao uso do dispositivo sônico. Ainda, deve-se investigar o efeito de dispositivos sônicos em adesivos autocondicionantes e em cimentos resinosos autoadesivos. 
Tabela 1. Análise descritiva dos valores de resistência de união (MPa) dos grupos avaliados

\begin{tabular}{|c|c|c|c|c|c|}
\hline Grupo & Mínimo & Máximo & Mediana & Média & Desvio Padrão \\
\hline 1 & 1,189 & 8,477 & $3,54906^{\AA}$ & 4,12180 & 2,364642 \\
\hline 2 & 0,612 & 5,308 & $2,35879^{\mathrm{B}}$ & 2,71259 & 1,384592 \\
\hline 3 & 1,131 & 7,534 & $2,48511^{\AA}$ & 3,40721 & 1,872656 \\
\hline
\end{tabular}

A,B Letras diferentes indicam valores estatisticamente significativos (teste de Mann Whitney; $p<0,05$ )

\section{Conclusão}

De acordo com os resultados do presente estudo, concluiu-se quea aplicação sônica do adesivo afetou os níveis de resistência de união do sistema Âmbar/AllCem à dentina exposta ao $\mathrm{NaOCl}$, atingindo valores significativamente semelhantes ao grupo sem $\mathrm{NaOCl}$. 


\section{Referências ::}

1. Haragushiku GA, Back ED, Tomazinho PH, BarattoFilho F, Furuse AY. Influence of antimicrobial solutions in the decontamination and adhesion of glass-fiber posts to root canals. J Appl Oral Sci. 2015;23:436-41.

2. Zehnder M. Root canal irrigants. J Endod. 2006;32:389-98.

3. Slutzky-Goldberg I, Maree M, Liberman R, Heling I. Effect of sodium hypochlorite on dentin microhardness. J Endod.2004;30(12):880-2.

4. Moreira DM, Almeida JF, Ferraz CC, Gomes BP, Line SR, Zaia AA.Structural Analysis of bovine root dentin after use of different endodontics auxiliary chemical substances. J Endod. 2009;35:1023-7.

5. Slutzky-Goldberg I, Maree M, Liberman R, Heling I. Effect of sodium hypochlorite on dentin microhardness. J Endod.2004;30(12):880-2.

6. Dantas MCC, Figueiredo IS, Pacheco LV, Prado M, Simão RA. Efeito da terapia de plasma de argônio em dentes expostos a hipoclorito de sódio: análise da hidrofilicidade. RFO. 2015;20:32-6.

7. Rasimick BJ, Wan J, Musikant BL, Deutsch AS.A review of failure modes in teeth restored with adhesively luted endodontic dowels. J Prosthodont. 2010;19:639-46.

8. Cagidiaco MC, Goracci C, Garcia-Godoy F, Ferrari M. Clinical studies of fiber posts: a literature review. Int J Prosthodont. 2008;21:328-36.

9. Cuadros-Sanchez J, Szesz A, Hass V, Patzlaff RT, Reis A, Loguercio AD.Effects of sonic application of adhesive systems on bonding fiber posts to root canals.J Endod. 2014;40:1201-5.

10. Schwartz RS. Adhesive dentistry and endodontics. Part 2: bonding in the root canal system-the promise and the problems: a review. J Endod. 2006;32:1125-34

11. Mena-Serrano A, Garcia EJ, Loguercio AD, Reis A. Effect of sonic application mode on the resin-dentin bond strength and nanoleakage of simplified self-etch adhesive. Clin Oral Investig. 2014;18:729-36.

12. Morris MD, Lee KW, Agee KA, Bouillaguet S, Pashley DH. Effects of
Sodium Hypochlorite and RC-Prep onBond Strengths of Resin Cement to Endodontic Surfaces.J Endod.2001;27:753-7.

13. Costa Dantas MC, do Prado M, Costa VS, Gaiotte MG, Simão RA, Bastian FL. Comparison between the effect of plasma and chemical treatments on fiber post surface. J Endod.2012;38:215-8.

14. Ferreira R, Prado M, de Jesus Soares A,Zaia AA, de Souza-Filho FJ.Influence of using clinical microscope as auxiliary to perform mechanical cleaning of post space: A bond strength analysis. J Endod. 2015;41:1311-6.

15. Sá TCM, Akaki E, Sá JCM. Esthetic post: which is the Best system? Arqu bras odontol. 2010;6:179-84.

16. Saunders WP.The shear impact retentive strengths of four dentine bonding agents to human and bovine dentine. J Dent. 1988;16:233-8.

17. Orstavik D, Haapasalo M. Disinfection by endodontic irrigants and dressings of experimentally infected dentinal tubules. EndodDentTraumatol. 1990;6:142-9.

18. Irma Cunha Matos IC, Sab TBB, Juliboni NC, Guerra RF, de Miranda MS. Utilização de dentes bovinos como possível substituto aos dentes humanos nos testes in vitro: revisão de literature. UFES Rev Odontol. 2008;10:58-63.

19. Hayashi M, Takahashi Y, Imazato S, Ebisu S. Fracture resistance of pulpless teeth restored with post- cores and crowns. Dent Mat.2006,22:477-85.

20. Santos JN, de Oliveira Carrilho MR, de Goes MF, Zaia AA, Gomes BP, Souza-Filho FJ, et al. Effect of chemical irrigants on the bond strength of a self-etching adhesive to pulp chamber dentin. J Endod. 2006;32:1088-90.

21. Farina AP, Cecchin D, Barbizam JV, Carlini-Junior B. Influence of endodontic irrigants on bond strength of a self-etching adhesive. AustEndod J.2011;37:26-30.

Recebido em: 17/05/2016 / Aprovado em: 12/06/2016

\section{Maira Prado}

Cidade Universitária- Centro de Tecnologia - Bloco F, sala F-201 - Ilha do Fundão

Rio de Janeiro/RJ, Brasil - CEP 21941-972

Email: maira@metalmat.ufrj.br

\section{Agradecimentos}

Os autores agradecem à empresa FGM pela doação dos pinos, adesivo, cimento e dispositivo sônico. Também agradecemos à Capes (PNPD) e CNPq (PIBIC) pelo apoio financeiro. 